\title{
The relationship between flux emergence and subsurface toroidal magnetic flux
}

\author{
R. H. Cameron ${ }^{1}$ and J. Jiang ${ }^{2}$ \\ 1 Max-Planck-Institut für Sonnensystemforschung, Justus-von-Liebig-Weg 3, 37077 Göttingen, Germany \\ e-mail: cameron@mps .mpg. de \\ 2 School of Space and Environment, Beihang University, Beijing 100083, PR China
}

Received 13 December 2018 / Accepted 8 September 2019

\begin{abstract}
Aims. The 1D mean-field equation describing the evolution of the subsurface toroidal field can be used with the observed surface radial field to model the subsurface toroidal flux density. Our aim is to test this model and determine the relationship between the observationally inferred surface toroidal field (as a proxy for flux emergence), and the modelled subsurface toroidal flux density. Methods. We used a combination of sunspot area observations and the surface toroidal field inferred from Wilcox Solar Observatory (WSO) line-of-sight magnetic field observations. We then compared them with the results of a 1D mean-field evolution equation for the subsurface toroidal field, driven by the observed radial field from the National Solar Observatory/Kitt Peak and SOLIS observations. Results. We derive calibration curves relating the subsurface toroidal flux density to the observed surface toroidal field strengths and sunspot areas. The calibration curves are for two regimes, one corresponding to ephemeral region emergence outside of the butterfly wings, the other to active region emergence in the butterfly wings. We discuss this in terms of the size and vertical velocity associated with the two types of flux emergence.
\end{abstract}

Key words. Sun: magnetic fields

\section{Introduction}

The most visible manifestations of the solar dynamo are sunspots. These appear at latitudes of about $\pm 30^{\circ}$ at the beginning of a solar activity cycle, and progressively closer to the equator as the solar cycle progresses to latitudes of about $\pm 8^{\circ}$ (Spoerer 1889) 11 years later (Schwabe 1849). Sunspots appear as part of the flux emergence process, where a magnetic field rises through the photosphere from below in bipolar active regions (for a review see Cheung \& Isobe 2014). The east-west orientation of the two polarities of active regions mostly (about 95\% for large active regions) follows Hale's law (Hale et al. 1919), indicating that they originate from the subsurface toroidal field of the global solar dynamo (for a review of dynamo theory see Charbonneau 2010, 2014).

The solar dynamo is thought to be an alpha-omega dynamo. This means the subsurface toroidal magnetic field is mainly generated by differential rotation acting on the poloidal magnetic field. In addition to the generation by differential rotation, the toroidal field is affected by the large-scale meridional flow, and small-scale turbulent convective eddies. The combined effect of these flows on the azimuthal toroidal flux can be described using mean-field magnetohydrodynamics (for an example, see Krause \& Rädler 1980). The well-known 1D mean-field equation for the toroidal field is discussed in Sect. 2. The ability of this $1 \mathrm{D}$ equation to describe the evolution of the toroidal field can be tested in conjunction with a few additional assumptions. These assumptions are that the poloidal field corresponds to the radial field at the surface, and that the rate at which systematically east-west oriented flux emerges is related to the amount of subsurface toroidal field. These latter assumptions are part of the
Babcock-Leighton model (some variants of which fall within the mean-field formalism).

In this paper, we investigate whether the 1D mean field equation (with the assumption that the observed surface radial field determines the poloidal flux) provides a good description of the field evolution. We do so by comparing the observed toroidal flux in the photosphere as a measure of flux emergence (Cameron et al. 2018), as inferred from Wilcox Solar Observatory (WSO) full-disk observations, with the subsurface toroidal field predicted from the evolution equation for subsurface toroidal field, and surface radial field measurements inferred from the Kitt Peak National Solar Observatory (NSO) and Synoptic Optical Long-term Investigations of the Sun (SOLIS) observations. The comparison is done both for active region emergences in the butterfly wings, and ephemeral region emergence in the quiet Sun.

Flux emergence occurs in discrete events. For example, the emergence of a large active region occurs over a few days and ranges in latitude from a few degrees and up to ten degrees in longitude. There is, from the point of view of a mean-field model, a considerable randomness in the details of the latitudes and timing of the flux emergence events. This affects our comparison: we expect some scatter due to the random nature of the flux emergence process. However, we demonstrate that taking yearly averages and latitudinal averages over several degrees is sufficient for the systematic component to be well-observed.

The paper is organised as follows: in Sect. 2, we present the WSO and NSO/SOLIS observations as well as the model. We compare the results of the model driven with the surface observations of the radial surface field with the observed toroidal field in Sect. 3. We present our conclusions in Sect. 4. 


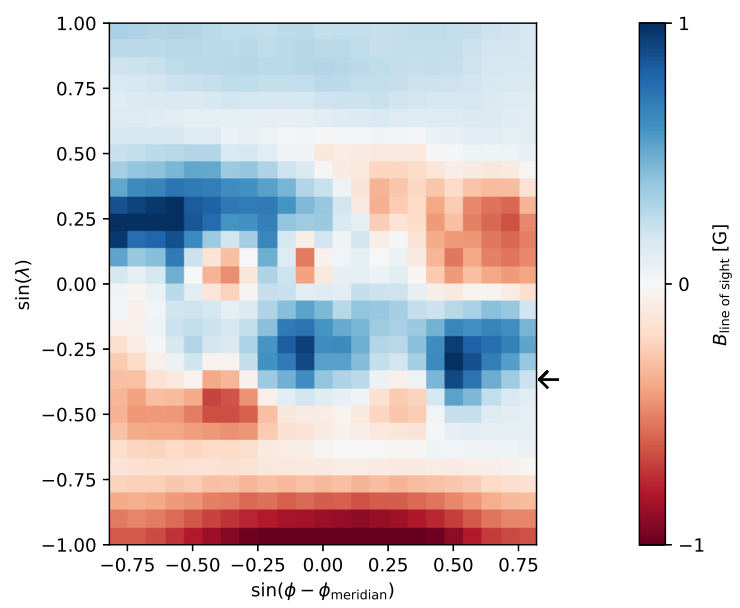

Fig. 1. Yearly averaged (6 September 2015-5 September 2016) lineof-sight magnetic field as function of latitude and longitude relative to central meridian $\left(\phi-\phi_{\text {meridian }}\right)$. The arrow indicates the latitude used in Fig. 2, where we illustrate the fitting procedure.

\section{Methods}

The surface toroidal (here meaning the azimuthally-averaged azimuthal) magnetic field corresponds to flux emergence, and can be inferred from observations (Cameron et al. 2018). As explained in that paper, the observed surface toroidal field results from flux emergence, where the horizontal magnetic field is carried across the solar surface. The amount of toroidal field observed at the surface is proportional to the flux emergence rate, with the constant of proportionality depending on the geometric properties of the emergence events, and the rise velocity of the flux through the photosphere. The geometric properties and rise velocity differ substantially between active regions and ephemeral regions, thus we analysed the activity wings (where active regions emerge) and outside the activity wings (where only ephemeral regions emerge) separately.

The procedure for determining the surface toroidal field is set out in Duvall (1978), Duvall et al. (1979) and Cameron et al. (2018). The starting point is the 40 years of WSO full disk magnetograms. In the current study, we divided the magnetograms into 12-month series from the 6 of September each year to the 5 of September of the following year (the dates chosen correspond to the maximum of the solar $B$ angle). The magnetograms for each 12-month period were used to create the average magnetogram as seen from the Earth, and an example of the average magnetogram is shown in Fig. 1. For each year and latitude, we then have a series of measurements of the line-of-sight field as a function of longitude with respect to the central meridian, an example of which is shown in Fig. 2. We fitted the 12-month average observed line-of-sight field for each latitude using

$B_{\mathrm{los}}=-B_{\phi} \sin \left(\phi-\phi_{\text {meridian }}\right)+B_{\mathrm{pol}} \cos \left(\phi-\phi_{\text {meridian }}\right)$,

where $B_{\phi}$ is the inferred toroidal field, and $B_{\text {pol }}$ the inferred lineof-sight component of the poloidal field. The error bars of each fit are inferred from the scatter in the data as a function of $\phi-$ $\phi_{\text {meridian }}$. The upper panel of Fig. 3 shows the butterfly map of the toroidal field constructed in this way.

In addition to the observed surface toroidal field, we needed to model the subsurface toroidal field. For this we used the 1D mean field equation describing the evolution of the toroidal field under the assumption that the winding up of the field differential rotation (the omega effect) dominates the alpha effect in the production of toroidal flux. This is likely to be the case with the Sun

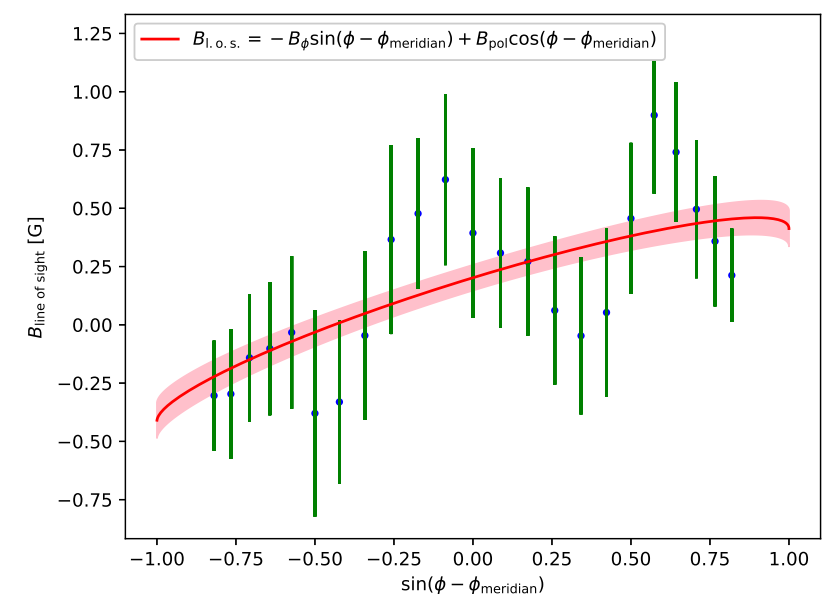

Fig. 2. Yearly averaged (6 September 2015-5 September 2016) line-ofsight magnetic field at latitude $-21^{\circ}$ (blue dots). The green-curves show the error estimate based on the scatter in the daily value going into the 12-month averaging. The red curve shows the fit $B_{\text {l.o.s. }}=-B_{\phi} \sin (\phi-$ $\left.\phi_{\text {meridian }}\right)+B_{\text {pol }} \cos \left(\phi-\phi_{\text {meridian }}\right)$, and the pink shaded region shows the $1-\sigma$ error estimates for the parameter fits.
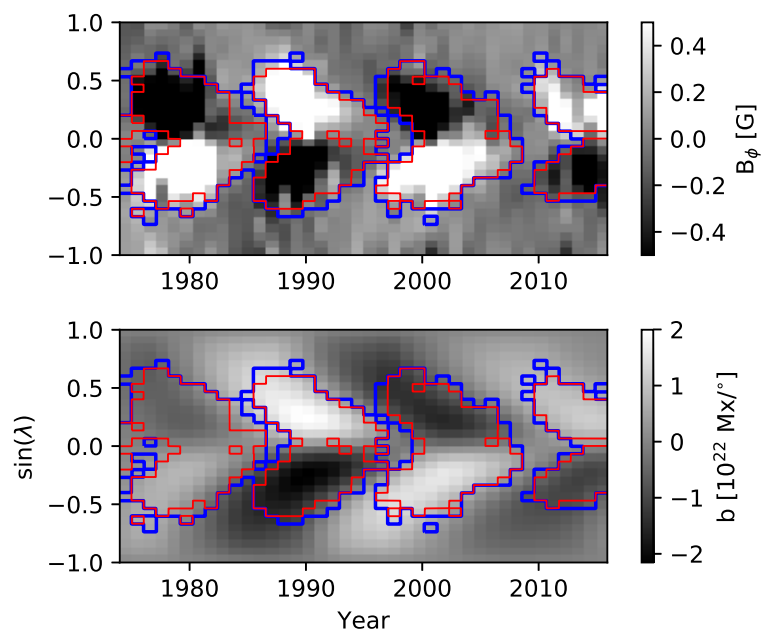

Fig. 3. Upper panel: azimuthally averaged surface toroidal magnetic field $\left(B_{\phi}\right)$ as a function time and latitude, saturated at $\pm 0.5 \mathrm{G}$. The maps are inferred from WSO full-disk magnetograms as described in the text. The blue curve outlines the pixels (times and latitudes) where any sunspots were reported in the RGO and SOLIS sunspot records. The red curves outline the region where the average coverage by sunspots exceeds $0.1 \%$ micro Hemispheres over the pixel. Lower panel: subsurface toroidal flux density based on model and observed surface radial field. The red and blue curves are the same as in the upper panel. We use the interior of the red curve as the butterfly wings and the exterior of the blue contour as the quiet-Sun.

(for example see Charbonneau 2010). We used the form of this equation given in Cameron \& Schüssler (2017) (which is appropriate when the effect of the poloidal field is determined by the surface radial field):

$$
\begin{aligned}
\frac{\partial b}{\partial t}= & \left.\sin \theta R_{\odot}^{2} B_{r}\right|_{R_{\odot}} \epsilon\left(\Omega_{R_{\odot}}-\Omega_{R_{\mathrm{NSSL}}}\right) \\
& -\left.\frac{\partial \Omega_{R_{\mathrm{NSSL}}}}{\partial \theta} \int_{0}^{\theta} \sin \theta R_{\odot}^{2} B_{\mathrm{r}}\right|_{R_{\odot}} \mathrm{d} \theta \\
& -\frac{1}{R_{\odot}} \frac{\partial}{\partial \theta}\left(V_{\theta} b\right)+\frac{\eta_{0}}{R_{\odot}^{2}} \frac{\partial}{\partial \theta}\left[\frac{1}{\sin \theta} \frac{\partial}{\partial \theta}(\sin \theta b)\right],
\end{aligned}
$$




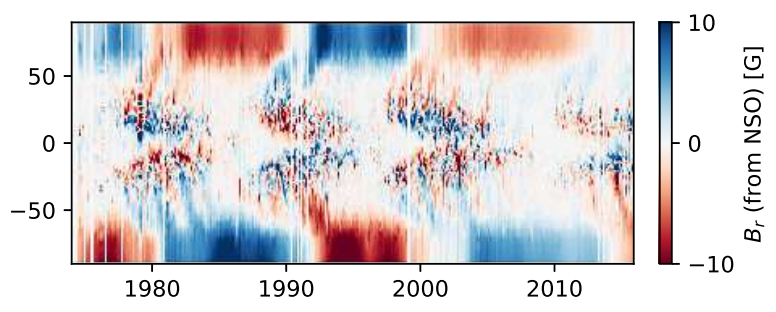

Fig. 4. Azimuthally averaged radial field from NSO/Kitt Peak and SOLIS observations as functions of latitude and time.

where $b=\int B_{\phi} r \mathrm{~d} r$ is the subsurface toroidal flux density, $\Omega_{R_{\odot}}$ and $\Omega_{R_{\text {NSSL }}}$ are the solar rotation rates at the solar surface. At the base of the near-surface shear layer, respectively, $\epsilon$ is a number between zero and one $(\epsilon=1$ if the field lines turn over at the base of the near-surface shear layer, and is smaller if the field lines turn over deeper where the solar rotation rate is smaller), $V_{\theta}$ is the radially averaged $\theta$ component of subsurface meridional flow (where the average is weighted by the radial distribution of the toroidal flux). It can in principle include both the bulk flow and the effect of latitudinal magnetic pumping. $\left.B_{\mathrm{r}}\right|_{R \odot}$ is the observed radial field at the solar surface, and $\eta_{0}$ is the turbulent magnetic diffusivity. For the subsurface meridional flow, we assumed the form $V_{\theta}=V_{0} \sin (2 \theta)$, where $V_{0}$ is a free parameter of the model. The meaning of the parameters and the sensitivity of the calculations to those parameters is discussed in Cameron \& Schüssler (2017), in which a parameter study was made. In this paper, we concentrated on the case with $V_{0}=2.5 \mathrm{~m} \mathrm{~s}^{-1}$ and $\eta_{0}=100 \mathrm{~km}^{2} \mathrm{~s}^{-1}$ and $\epsilon=1$ (we found similar results with a range of other values of the parameters).

It is to be noted that Eq. (2) corresponds to one of the two equations of the updated Babcock-Leighton model, exactly because the evolution of the toroidal field in the BabcockLeighton model is that of the mean-field description of an alphaomega dynamo. The equation is also that of a flux transport dynamo, when the latitudinal velocity $V_{\theta}$ plays a substantial role. This flow corresponds to a combination of the equatorward subsurface return flow (the counterpart to the well-observed surface meridional flow) and the subsurface latitudinal pumping, which is not observationally constrained. For a 1D model, these are weighted radial averages with a weighting corresponding to the unknown radial distribution of the subsurface toroidal field. These considerations result in the flow being currently poorly constrained by observations. The flow is expected, based on symmetry arguments, to vanish at the equators and poles (on average) which is the basis for the assumption of the $\sin (2 \theta)$ latitudinal form. The amplitude is a free parameter of the model, and the value chosen has previously been used to qualitatively reproduce the butterfly diagram. The sign of $V_{\theta}$ corresponds to equatorial propagation.

We drived the evolution of the subsurface toroidal field using observations: Eq. (2) requires knowledge of the surface radial field, for which we used NSO/Kitt Peak and SOLIS observations as presented by Petrie \& Ettinger (2017), shown in Fig. 4. The resulting model subsurface toroidal field mapped to the spatial and temporal resolution of the observed surface toroidal field is shown in the lower panel of Fig. 3.

\section{Results}

The observed surface toroidal field and subsurface toroidal flux density are shown in Fig. 3. We divide the two images into the butterfly wings and quiet-Sun regions based on sunspot
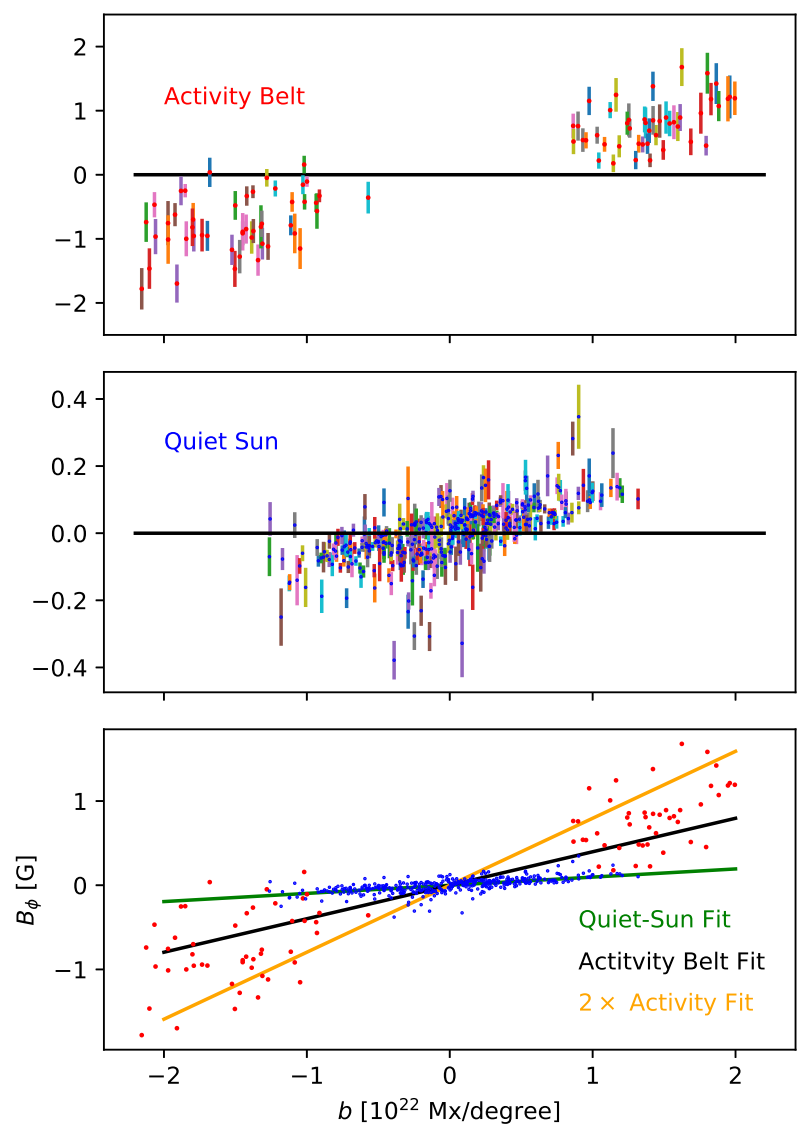

Fig. 5. Pixel-by-pixel comparison of subsurface toroidal flux density from model $b$, and observed surface toroidal field $B_{\phi}$, for cycles 22,23 and 24 . We excluded cycle 21 from this analysis as it is heavily affected by the initial condition $b=0$. Upper panel: results for the butterfly wings where flux emergence includes active region emergences. Middle panel: results for the quiet-Sun where the emergences are in the form of ephemeral regions. Bottom panel: both types of regions. The first two panels also show the error bars on the observed surface toroidal field obtained from the fitting procedure, in different colours to make them easier to distinguish. In the bottom panel we also show fits for both the quiet-Sun and butterfly wing data.

coverage $^{1}$. We now consider a pixel by pixel comparison of the toroidal surface field and subsurface flux density for both the butterfly wings and the quiet-Sun, with the result shown in Fig. 5.

Qualitatively, the upper two panels of Fig. 5 show that both inside and outside of the butterfly wings, the variability of the surface toroidal field is partly captured by the model - a clear, approximately linear trend is visible. We also see that the butterfly wings mostly correspond to subsurface toroidal flux densities of over $10^{22} \mathrm{Mx} \mathrm{deg}^{-1}$. As a threshold subsurface flux density for the formation of sunspots, this should be compared to the range of fluxes of bipolar active regions with sunspots of $5 \times 10^{21}$ $3 \times 10^{22}$ (Table 5.1 of Schrijver \& Zwaan 2008) and to a spatial scale of a few degrees. The threshold of $10^{22} \mathrm{Mx} \mathrm{deg}^{-1}$ is thus higher than what is strictly necessary to account for the amount of flux which emerges, but only by a factor of a few, and might reflect the fact that not all the flux available beneath the surface emerges during each emergence event. The next point to note is that the average observed surface toroidal field increases with the subsurface toroidal flux density, but there is a large amount

\footnotetext{
1 The sunspot area data were obtained from https:// solarscience.msfc.nasa.gov/greenwch.shtml
} 
of scatter. Quantitatively, we choose to fit the relationship for the butterfly wings with a function of the form $B_{\phi}=a_{\text {wings }} b$, where $a_{\text {wings }}=4.0 \times 10^{-23} \pm 0.1 \times 10^{-23} \mathrm{G}\left(\mathrm{Mx} \mathrm{deg}^{-1}\right)^{-1}$ is the fitted parameter. The quality of the fit is poor, $\chi^{2}=501$ for the 93 data pairs (with $\left.\chi^{2} /(93-1)=5.4\right)$. This indicates that the model does not fully explain the surface toroidal field. This presumably reflects a combination of problems with the model, and the fact that the apparently partly stochastic flux emergence introduces a substantial randomness into $B_{\phi}$.

For the points in the butterfly wings we also performed a fit to

$B_{\phi} \operatorname{sign}(b)=a|b|+c$,

where $a$ and $c$ are the two fit parameters. The idea was to test if the linear fit mainly shows that the model gets the sign of $b$ correct (i.e. the same sign as $B_{\phi}$ ). Fitting to Eq. (3) allows a non-zero $c$, even in the event that the model gets the sign correct. However, we find that the fit is $a=3.9 \times 10^{-23} \pm 0.5 \times 10^{-23} \mathrm{G}\left(\mathrm{Mx} \mathrm{deg}^{-1}\right)^{-1}$, and $c=0.04 \times 10^{-23} \pm 0.6 \times 10^{-23} \mathrm{G}\left(\mathrm{Mx} \mathrm{deg}^{-1}\right)^{-1}$, so $c$ is small and consistent with zero. Therefore, we conclude that the linear fit does not simply show the model getting the sign of $b$ correct.

In the case of the quiet-Sun, the underlying subsurface toroidal flux density is below $1.25 \times 10^{22} \mathrm{Mx} \mathrm{deg}^{-1}$, and the fit of the surface toroidal field is $B_{\phi}=a_{\mathrm{qs}} b$ with $a_{\mathrm{qs}}=0.97 \times$ $10^{-23} \pm 0.015 \times 10^{-23} \mathrm{G}\left(\mathrm{Mx} \mathrm{deg}^{-1}\right)^{-1}$. Hence the range of subsurface toroidal fluxes in the quiet-Sun partly overlapping with those of the butterfly wings. The quality of the fit is again poor, with $\chi^{2}=2964$ for 382 data pairs (with $\chi^{2} /(382-1)=7.7$ ), again presumably reflecting a combination of problems with the model and the random component to flux emergence.

The fits for the quiet-Sun and butterfly wings are very different, $a_{\mathrm{qs}}=0.97 \times 10^{-23} \pm 0.015 \times 10^{-23} \mathrm{G}\left(\mathrm{Mx} \mathrm{deg}^{-1}\right)^{-1}$ versus $a_{\text {wings }}=4.0 \times 10^{-23} \pm 0.1 \times 10^{-23} \mathrm{G}\left(\mathrm{Mx} \mathrm{deg}^{-1}\right)^{-1}$. A substantial part of this is presumably related to the rise speed of the ephemeral regions and active regions through the photosphere. As discussed in Cameron et al. (2018), the surface toroidal field corresponds to the horizontal field being carried through the surface during flux emergence. The impact of the horizontal field depends on how long it takes to traverse the photosphere, which is inversely proportional to the vertical velocity at the surface. Ephemeral regions rise through the photosphere more quickly, and thus we expect $a_{\mathrm{qs}}$ to be smaller than $a_{\text {wings. }}$. The degree to which we expect $a_{\mathrm{qs}}$ to be smaller is difficult to estimate, because not all the flux that emerges in the butterfly wings is in the form of active regions. The point of this paragraph is to state that the difference between $a_{\mathrm{qs}}$ and $a_{\text {wings }}$ does not necessarily indicate a difference in the subsurface dynamics of the two classes of emergences.

The surface toroidal field corresponding to a given subsurface flux density can then be modelled as

$B_{\phi}= \begin{cases}a_{\mathrm{qs}} \times b & \text { if }|b|<10^{22} \mathrm{Mx} \mathrm{deg}^{-1} \\ a_{\mathrm{wings}} \times b & \text { otherwise. }\end{cases}$

In order to relate the subsurface toroidal flux to the sunspot number, we apply a threshold found above of $10^{22} \mathrm{Mx} \mathrm{deg}^{-1}$. We then integrate the flux above this threshold in each hemisphere as a function of time and compare this with the sunspot area coverage in each hemisphere. The model has no sunspots appearing during cycle 21 , because we began on the (obviously incorrect) initial condition that $b=0$ in 1974. The model has a memory of the initial condition of at least one cycle. The maxima of cycles 22,23 and 24 are partly reproduced.
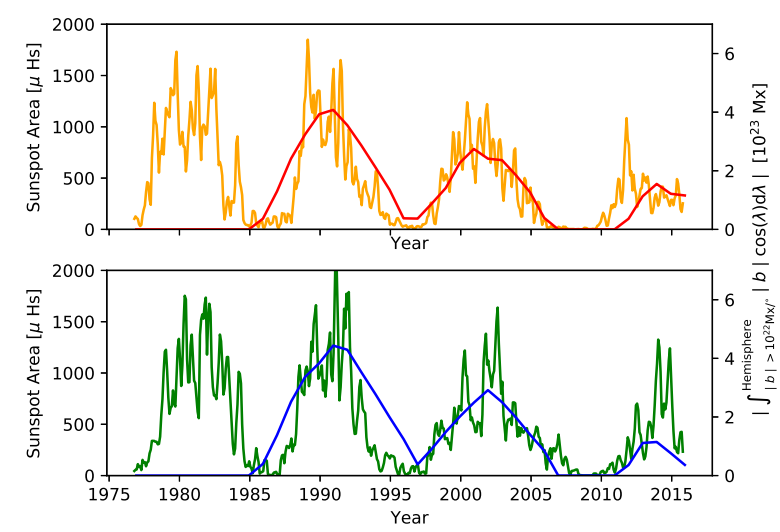

Fig. 6. Sunspot coverage in northern (southern) hemisphere as function of time in orange (green). Also shown is the integral of the subsurface toroidal flux density in each hemisphere where it exceeds $10^{22} \mathrm{Mx} \mathrm{deg}^{-1}$.

\section{Conclusion}

In this paper we investigated the relationship between the surface toroidal field (and sunspot area coverage) as a function of the subsurface toroidal field as predicted by the surface radial field measurements applied to the 1D mean-field evolution equation for the subsurface toroidal flux density with a poloidal field derived from the surface radial magnetic field observations.

We find that the fit between the model subsurface flux density and the surface toroidal field differs in the quiet-Sun and in the butterfly wings, which we suggest might be because of the different speeds at which ephemeral regions and active regions emerge through the photosphere. In both cases we find that the quality of the fit as measured by the $\chi^{2}$ statistic is relatively poor, possibly because flux emergence introduces a random component.

Our results allow the subsurface toroidal flux density of the model to be quantitatively related to observable quantities such as sunspot areas and surface toroidal fields.

Acknowledgements. J. J. acknowledges the support by the National Science Foundation of China (grant Nos. 11873023, 11522325) and by the Fundamental Research Funds for the Central Universities of China. Wilcox Solar Observatory data used in this study was obtained via the web site http://wso. stanford. edu, courtesy of J. T. Hoeksema. NSO/Kitt Peak data used here are produced cooperatively by NSF/NOAO, NASA/GSFC, and NOAA/SEL. This work utilizes SOLIS data obtained by the NSO Integrated Synoptic Program (NISP), managed by the National Solar Observatory, which is operated by the Association of Universities for Research in Astronomy (AURA), Inc. under a cooperative agreement with the National Science Foundation. We thank Manfred Schüssler and two referees for useful comments.

\section{References}

Cameron, R. H., \& Schüssler, M. 2017, A\&A, 599, A52

Cameron, R. H., Duvall, T. L., Schüssler, M., \& Schunker, H. 2018, A\&A, 609, A56

Charbonneau, P. 2010, Liv. Rev. Sol. Phys., 7, 3

Charbonneau, P. 2014, ARA\&A, 52, 251

Cheung, M. C. M., \& Isobe, H. 2014, Liv. Rev. Sol. Phys., 11, 3

Duvall, Jr., T. L. 1978, PhD Thesis, Stanford Univ., CA

Duvall, Jr., T. L., Scherrer, P. H., Svalgaard, L., \& Wilcox, J. M. 1979, Sol. Phys., 61,233

Hale, G. E., Ellerman, F., Nicholson, S. B., \& Joy, A. H. 1919, ApJ, 49, 153

Krause, F., \& Rädler, K.-H. 1980, Mean-field Magnetohydrodynamics and Dynamo Theory (Berlin: Akademie-Verlag)

Petrie, G., \& Ettinger, S. 2017, Space Sci. Rev., 210, 77

Schrijver, C. J., \& Zwaan, C. 2008, Solar and Stellar Magnetic Activity (Cambridge, UK: Cambridge University Press)

Schwabe, M. 1849, Astron. Nachr., 28, 302

Spoerer, G. 1889, Nova Acta der Ksl. Leop.- Carol. Deutschen Akademie der Naturforscher, 53, 281 